\title{
Assessment of phylogenetic structure in genome size - gene content correlations
}

\author{
Prasad, V R ; Isler, K
}

\begin{abstract}
Gene content and gene-coding percentage can be predicted from genome size in newly sequenced organisms. Here, we investigate whether these predictions are influenced by phylogenetic relationships between the involved species. Combining a highly resolved phylogenetic tree with a large compilation of gene content data, our results reveal the presence of significant phylogenetic structure in the correlations between genome size and gene content in both bacteria and eukaryotes. The variation in $\log$ (gene content) explained by $\log$ (genome size) in combination with phylogeny was found to be $97 \%$ in bacteria and $55 \%$ in eukaryotes. Further, in bacteria, gene-coding percentages are only significantly correlated to genome size if phylogenetic information is taken into account in the analyses. These findings support the usage of phylogenetic correlation models for gene content predictions.
\end{abstract}

DOI: https://doi.org/10.1139/g2012-019

Posted at the Zurich Open Repository and Archive, University of Zurich

ZORA URL: https://doi.org/10.5167/uzh-70279

Journal Article

Accepted Version

Originally published at:

Prasad, V R; Isler, K (2012). Assessment of phylogenetic structure in genome size - gene content correlations. Genome (Génome), 55(5):391-395.

DOI: https://doi.org/10.1139/g2012-019 
1 Assessment of phylogenetic structure in genome size-gene content correlations

2

3

$4{ }^{1}$ Institute of Molecular Life Sciences, University of Zurich-Irchel, Winterthurerstrasse 190, $5 \quad \mathrm{CH}-8057$ Zurich, Switzerland

6

7

8

9

10

\section{NOTE}

\section{Vibhu Ranjan Prasad ${ }^{1}$ and Karin Isler $^{2}$}

2 Anthropological Institute and Museum, University of Zurich-Irchel, Winterthurerstrasse 190, CH-8057 Zurich, Switzerland

Published in: Genome (2012) 55, p. 391-395

\section{Corresponding author:}

Vibhu Ranjan Prasad, Institute of Molecular Life Sciences, University of Zurich-Irchel, Winterthurerstrasse 190, CH-8057 Zurich, Switzerland

phone/fax: $+41(0) 446355396$

email: vibhuranjan.prasad@,uzh.ch

Keywords: genome size, gene content, gene-coding percentage, phylogeny, independent contrasts

Running head: Phylogeny predicts genome size-gene content correlation

\section{List of abbreviations:}

IC: independent contrasts

PGLS: phylogenetic least-squares regression 


\section{ABSTRACT}

2 Gene content and gene-coding percentages can be predicted from genome size in newly

3 sequenced organisms. Here, we investigate whether these predictions are influenced by

4 phylogenetic relationships between the involved species. Combining a highly resolved

5 phylogenetic tree with a large compilation of gene content data, our results reveal the

6 presence of significant phylogenetic structure in the correlations between genome size and

7 gene content in both bacteria and eukaryotes. The variation in log-gene content explained by

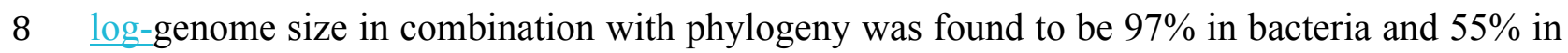

9 eukaryotes. Further, in bacteria gene-coding percentages are only significantly correlated to

10 genome size if phylogenetic information is taken into account in the analyses. These findings

11 support the usage of phylogenetic correlation models for gene content predictions. 


\section{TEXT}

2 Gene content, the number of genes coding for proteins, is correlated with genome size in both

3 non-eukaryotes and eukaryotes (Lynch and Conery 2003; Konstantinidis and Tiedje 2004;

4 Gregory 2005). More detailed knowledge about this relationship would be important, as it is

5 highly desirable to be able to predict gene content in species with massive genome size.

6 Recently, Hou and Lin (2009) proposed a linear relationship between $\log$ (genome size) and

$7 \log ($ gene content) for non-eukaryotes and a steeper logarithmic relationship for eukaryotes,

8 both with high correlation of $\underline{R}^{2}>0.91$. Similar relationships were found between genome size

9 and the total number of genes, or, inversely, with the amount of non-coding DNA. Their compilation includes 55 eukaryote and 1055 non-eukaryote species with completely

sequenced and annotated genomes. However, given that these species are phylogenetically related to various degrees, phylogenetic information should be included in the correlation analyses, which has not been attempted yet. Here, we examine the correlations between genome size and gene content or coding percentage of genome, applying methods for "phylogenetically informed analysis" that have been developed for correlative studies in other fields (e.g. Felsenstein 1985; Garland et al. 2005), The method of phylogenetic generalized least-squares (PGLS) is currently the most frequently used approach for phylogenetic regression (Nunn 2011), as it allows the assumed model of evolution to deviate from Brownian motion (Pagel 1999; Freckleton et al. 2002). To test for phylogenetic structure in $\underline{\text { the data, PGLS simultaneously estimates the parameter } \lambda \text { for a given combination of data and }}$ $\underline{\text { tree with a maximum likelihood approach. A } \lambda \text {, value significantly different from } 0 \text { indicates }}$ $\underline{\text { that the data values cluster according to the structure of the given phylogenetic tree, and thus }}$ that phylogenetic regression is warranted. If $\lambda$ is close to 1 , PGLS yields roughly the same 
1 Phylogenetic and non-phylogenetic correlations between genome size and both gene-content

2 and gene-coding percentages are shown in Table 1 and illustrated in Figure 2. For the

3 correlation between gene-content and genome size, $\lambda$ values are close to 1 and significantly

4 different from 0 in both eukaryotes and non-eukaryotes, indicating that a phylogenetic

5 approach is needed for this analysis. Thus, results of phylogenetic least-squares regression

6 (PGLS) are almost identical to independent contrasts (IC) results in all three groups. Overall,

7 the correlation coefficients and p-values are very similar in phylogenetic vs. non-phylogenetic

8 analyses, although for eukaryotes, our analysis yields a weaker correlation in phylogenetic

9 regression $\left(\mathrm{R}^{2}=0.55, p=.0005\right)$ than in raw species regression $\left(\mathrm{R}^{2}=0.84, p<.0001\right)$. The

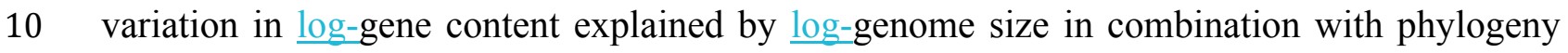

11 was found to be $97 \%$ in bacteria and $55 \%$ in eukaryotes.

12 For the correlation of gene coding percentage with genome size, $\lambda$ values are close to 1 and

13 differ significantly from zero for the bacterial and the combined dataset, but not for

14 eukaryotes (Table 1). Thus, PGLS results are more similar to raw correlations in the latter

15 group. Interestingly, the very low correlation between genome size and gene-coding 16 percentage in bacteria is only significantly different from zero if phylogenetic relationships 17 are taken into account. Log-genome size predicts $81 \%$ of the variation in log-gene-coding 18 percentage in eukaryotes, but only about $7 \%$ in bacteria (for prediction equations, see 19 Supplementary Information).

20 Our results indicate the presence of phylogenetic structure in the correlations of genome size 21 with gene content in both bacteria and eukaryotes, and in the correlation of genome size with 22 gene-coding percentage in bacteria. The absence of phylogenetic structure in eukaryote gene23 coding percentages may be due to the relatively small number of species in our sample $24(n=19)$, which are all very distantly related (e.g._1 bird, 1 fish, 1 worm, 1 algae and 4 25 mammals). In bacteria, the sample is larger $(n=82)$ and the variation in the degree of

\section{Vibhu}

Delet Vibhu Delet Vibhu Delet 
1 relationship between the included species is higher, yielding a better estimate of phylogenetic

2 structure. In contrast to earlier studies (Hou and Lin 2009), we found that the gene-coding

3 percentage is also significantly correlated to genome size in bacteria, but only if phylogenetic

4 relationships between species are taken into account in the analysis.

5 We therefore conclude that phylogenetic structure should be considered in any attempt to

6 predict gene content or gene-coding percentage from genome size in organisms. Although the

7 present sample is rather small, we expect that the amount of phylogenetic structuring will be even larger if the sample is expanded by inclusion of more, closely related species.

Additionally, considering phylogenetic relationships may provide insights into whether a

particular deviation from the general trend is species-specific (e.g. due to recent polyploidy,

cf. Otto 2007) or lineage-specific (e.g. an adaptive event in ancestral birds and bats, Hughes and Hughes 1995). Depending on the availability of larger datasets, future studies may disentangle different scenarios of phylogenetic inertia and adaptation in gene content genome size variation across organisms, as has proven useful in comparataive studies of genome size (e.g. Oliver et al. 2007, Organ and Shedlock 2009).

\section{METHODS}

Taken from Hou and Lin (2009), the variables in the present study include genome size, protein coding gene number, and coding percentage. All data were log-transformed in order to $\underline{\text { increase normality of their distributions over a large range of values, following Hou and Lin }}$

21 (2009). Phylogenetic information was taken from the Interactive Tree of Life (Letunic and 
1 phylogeny was constructed using Maximum Likelihood reconstruction as described in

2 Ciccarelli et al. (2006). This approach was chosen as it allows to include a very diverse range

3 of organisms in a consistent manner, although it may not yield a maximum sample size within

4 lineages. Limited by the overlap between the two, the dataset on gene content and the

5 phylogenetic tree, our sample comprises 101 organisms (82 bacteria and 19 eukaryotes listed

6 in the Supplementary Information). The pruned version of the tree used in this analysis is

7 shown in Figure 1.

9 To test whether there is phylogenetic autocorrelation in the data, and thus whether a

10 phylogenetic approach is warranted, the parameter $\lambda$ was estimated using the CAIC package

11 (Purvis and Rambaut 1995) in R 2.14 ( $\mathrm{R}$ Development Core Team 2011). Varying from 0 to

$121, \lambda$ close to 0 indicates that there is no phylogenetic signal in the data, whereas $\lambda$ close to 1

13 indicates Brownian motion phylogenetic autocorrelation in the analysed traits. In other words,

14 if the value of $\lambda$ is found to be significantly different from zero, phylogenetic methods such as

15 the here applied phylogenetic generalized least squares (PGLS) regression are warranted

16 (Pagel 1999). PGLS fits a linear model to the data, which therefore must be transformed

17 accordingly before analysis. Although Hou and Lin (2009) found a slightly better fit for a

18 logarithmic relationship between $\log ($ gene cont) and $\log$ (genome size), in our smaller sample

19 the difference between a logarithmic and a linear model was minimal. We therefore

20 consistently used a linear model for all analyses. PGLS converts the phylogeny into a

21 variance-covariance matrix, which is then included in the error term of the regression model.

22 The resulting estimated regression parameters are "phylogenetically controlled" (Pagel 1999;

23 Freckleton et al. 2002). For illustration, phylogenetically independent contrasts (IC.

24 Felsenstein 1985; Garland et al. 1992) were calculated in Mesquite (Maddison and Maddison 
1 2008) using the PDAP: PDTREE package (Midford et al. 2002) and analysed using the

2 program JMP (JMP 2009).

\section{Acknowledgements}

4 Vibhu Ranjan Prasad would like to thank Dhirendra Mohan Prasad, Anubhuti Ranjan Prasad

5 and Anand Mala Prasad for their continuous encouragement. We thank two anonymous

6 reviewers for their helpful comments on an earlier version of the manuscript.

7

8

9 Ciccarelli, F.D., Doerks, T., Von Mering, C., Creevey, C.J., Snel, B., Bork, P. 2006. Toward

\section{Literature cited} automatic reconstruction of a highly resolved tree of life. Science, 311:1283-1287.

Felsenstein, J. 1985. Phylogenies and the comparative method. Am. Nat. 125:1-15.

Freckleton, R.P., Harvey, P.H., Pagel, M. 2002. Phylogenetic analysis and comparative data: 3 A test and review of evidence. Am. Nat. 160:712-726.

Garland Jr, T., Bennett, A.F., Rezende, E.L. 2005. Phylogenetic approaches in comparative physiology. J. Exp. Biol. 208:3015-3035.

Garland, T., Harvey, P.H., Ives, A.R. 1992. Procedures for the analysis of comparative data using phylogenetically independent contrasts. Syst. Biol. 41:18-32.

Gregory, T.R. 2002. A bird's-eye view of the C-value enigma: genome size, cell size, and $\underline{\text { metabolic rate in the class Aves. Evolution, 56:121-130. }}$

Gregory, T.R. 2005. Synergy between sequence and size in large-scale genomics. Nat. Rev. Genet. 6:699-708. 
1 Hou, Y., Lin, S. 2009. Distinct gene number-genome size relationships for eukaryotes and non-eukaryotes: gene content estimation for dinoflagellate genomes. PLoS One, 4:e6978.

Hughes, A.L., Hughes, M.K. 1995. Small genomes for better flyers. Nature 377:391.

JMP 2009 . Version 8.0. SAS Institute. Inc., Cary, NC.

Konstantinidis, K.T., Tiedje, J.M. 2004. Trends between gene content and genome size in prokaryotic species with larger genomes. Proc. Natl. Acad. Sci. U.S.A. 101:31603165.

Letunic, I., Bork, P. 2007. Interactive Tree Of Life (iTOL): an online tool for phylogenetic tree display and annotation. Bioinformatics, 23:127-128.

Lynch, M., Conery, J.S. 2003. The origins of genome complexity. Science, 302:1401-1404.

Maddison, W.P., Maddison, D.R. 2008. Mesquite: a modular system for evolutionary analysis. Evolution, 62:1103-1118.

Midford, P., Garland Jr, T., Maddison, W. 2002. PDAP: PDTREE package for Mesquite, version 1.00. Available from http://www. http://mesquiteproject.org/pdap_mesquite/ [accessed 21 July 2011].

Nunn, C.L. 2011. The Comparative Approach in Evolutionary Anthropology and Biology. University of Chcago Press, Chicago, IL.

Oliver, M.J., Petrov, D., Ackerly, D., Falkowski, P, Schofield, O.M. 2007. The mode and tempo of genome size evolution in eukaryotes. Genome Res. 17:594-601.

Organ, C.L, Shedlock, A.M. 2009. Paleogenomics of pterosaurs and the evolution of small genome size in flying vertebrates. Biol. Letters, 5:47-50.

Otto, S.P. 2007. The evolutionary consequences of polyploidy. Cell, 131:452-462.

Pagel, M. 1999. Inferring the historical patterns of biological evolution. Nature, 401:877-884. 
1 Purvis, A., Rambaut, A. 1995. Comparative analysis by independent contrasts (CAIC): an

3 R Development Core Team, 2011. R: A language and environment for statistical computing.

$4 \quad$ R Foundation for Statistical Computing, Vienna, Austria Version 2.14.

7 Tables

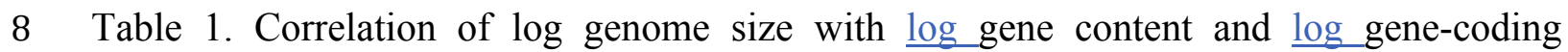

9 percentage for eukaryotes, bacteria and all species using phylogenetic generalized least-

10 squares (PGLS), a non-phylogenetic model (Raw) and independent contrasts (IC).

11 Regression equations are shown in the Supplementary Information.

\begin{tabular}{|c|c|c|c|c|c|c|c|c|c|c|}
\hline & \multicolumn{5}{|c|}{ Gene content } & \multicolumn{5}{|c|}{ Gene-coding percentage } \\
\hline & & PGLS & & $\begin{array}{l}\text { Raw } \\
(\lambda=0)\end{array}$ & $\begin{array}{c}\mathbf{I C} \\
(\lambda=1)\end{array}$ & & PGLS & & $\begin{array}{c}\text { Raw } \\
(\lambda=0)\end{array}$ & IC $(\lambda=1)$ \\
\hline & $\lambda$ & $\begin{array}{c}\text { P- } \\
\text { value } \\
\text { of } \lambda \neq 0\end{array}$ & $\begin{array}{c}\mathbf{R}^{2} \\
\mathbf{P}- \\
\text { value }\end{array}$ & $\begin{array}{r}\mathbf{R}^{2} \\
\text { P-value }\end{array}$ & $\begin{array}{c}\mathrm{R}^{2} \\
\text { P-value }\end{array}$ & $\lambda$ & $\begin{array}{c}\text { P- } \\
\text { value } \\
\text { of } \lambda \neq 0\end{array}$ & $\begin{array}{c}\mathbf{R}^{2} \\
\mathbf{P}- \\
\text { value }\end{array}$ & $\begin{array}{c}\mathrm{R}^{2} \\
\text { P-value }\end{array}$ & $\begin{array}{r}\mathbf{R}^{2} \\
\text { P-value }\end{array}$ \\
\hline $\begin{array}{c}\text { Eukaryote } \\
\qquad(n=19)\end{array}$ & 0.97 & $0.03 *$ & $\begin{array}{c}0.55 \\
.0002 *\end{array}$ & $\begin{array}{c}0.82 \\
<.0001^{*}\end{array}$ & $\begin{array}{c}0.51 \\
0.0005^{*}\end{array}$ & 0.001 & 1 & $\begin{array}{r}0.819 \\
<.0001^{*}\end{array}$ & $\begin{array}{c}0.819 \\
<.0001^{*}\end{array}$ & $\begin{array}{c}0.46 \\
0.003 * \\
\end{array}$ \\
\hline $\begin{array}{c}\text { Bacteria } \\
(n=82)\end{array}$ & 0.84 & $0.0007^{*}$ & $\begin{array}{c}0.97 \\
<.0001^{*}\end{array}$ & $\begin{array}{c}0.97 \\
<.0001^{*}\end{array}$ & $\begin{array}{c}0.96 \\
<.0001 *\end{array}$ & 0.999 & $<.0001 *$ & $\begin{array}{c}0.068 \\
0.017^{*}\end{array}$ & $\begin{array}{c}0.013 \\
0.29\end{array}$ & $\begin{array}{c}0.069 \\
0.016^{*}\end{array}$ \\
\hline $\begin{array}{c}\text { All } \\
\text { species } \\
(n=101)\end{array}$ & 0.97 & $<.0001^{*}$ & $\begin{array}{c}0.74 \\
<.0001 *\end{array}$ & $\begin{array}{r}0.798 \\
<.0001 *\end{array}$ & $\begin{array}{c}0.725 \\
<.0001^{*}\end{array}$ & 0.967 & $<.0001^{*}$ & $\begin{array}{r}0.264 \\
<.0001 * \\
\end{array}$ & $\begin{array}{r}0.77 \\
<.0001 *\end{array}$ & $\begin{array}{c}0.08 \\
0.003^{*}\end{array}$ \\
\hline
\end{tabular}




\section{Figure legends}

2 Fig 1. The highly resolved phylogenetic tree of 101 species used in this study for Independent

3 Contrasts and PGLS.

4 Fig 2. Correlations of $\log$ (gene content) and $\log$ (gene-coding percentage) with genome size without

5 phylogeny (A, C), and with phylogeny (B, D) using independent contrasts (IC). Dashed line is 6 eukaryotic and solid line is bacterial fit. For statistics see Table 1. 


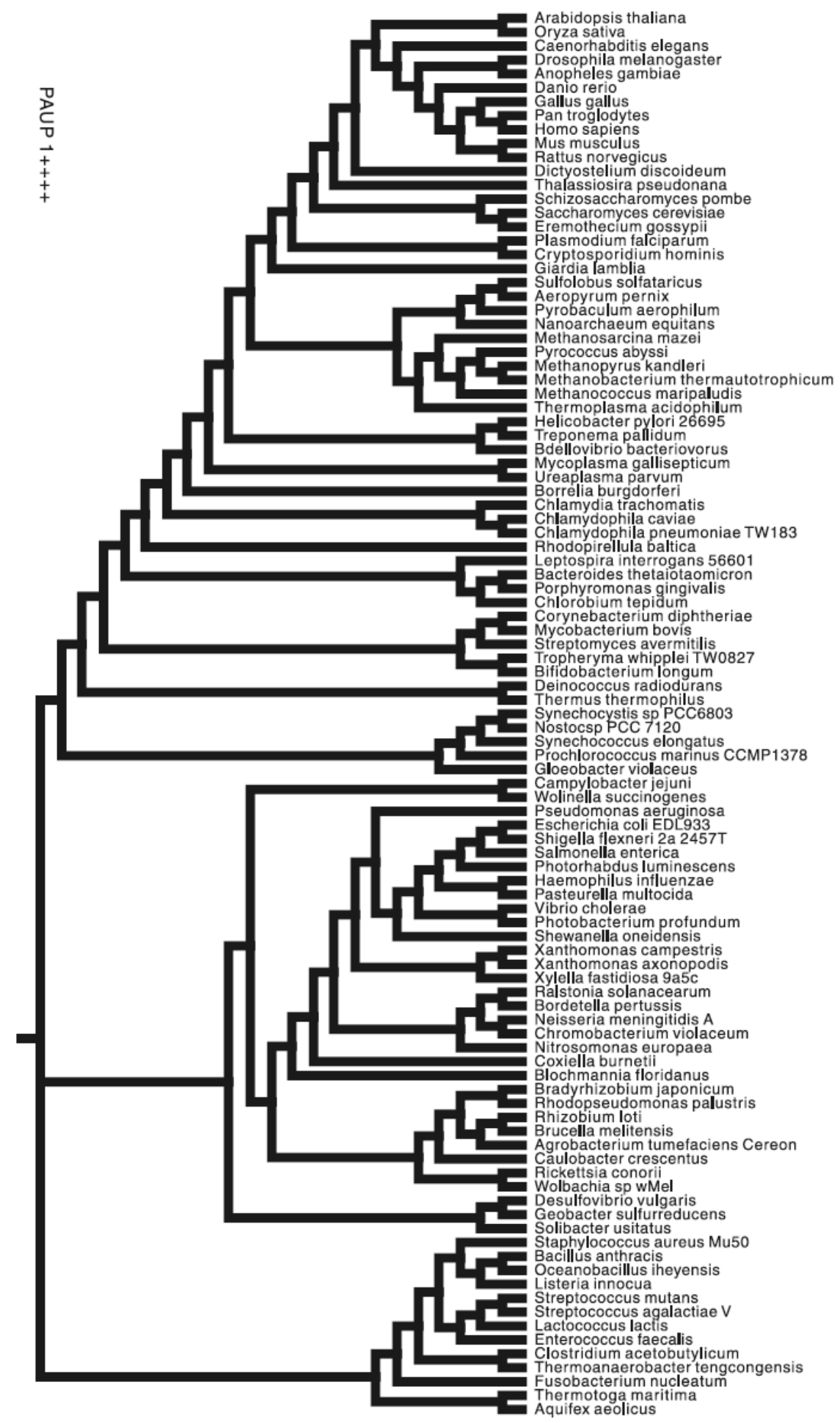



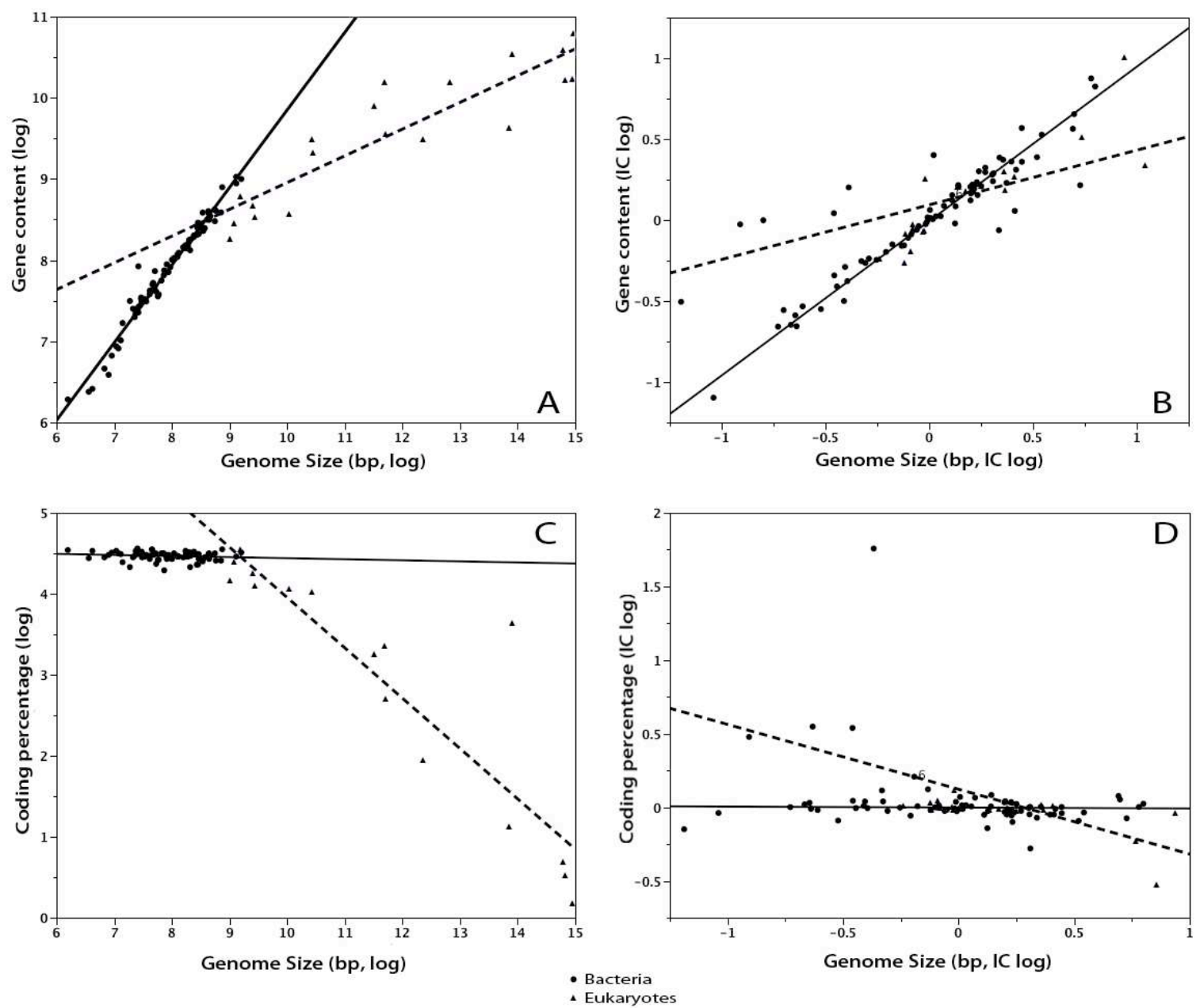